\title{
Chocolate and strawberries
}

\section{As genome sequencing becomes more versatile and easier, the journal prioritizes those genomic sequences that maximize the prospects of harnessing genome variation and understanding evolutionary processes.}

$T$ his month's issue is full of treats for lovers-and for lovers of genomics. We present the first reference genomes of the Criollo cocoa variety Theobroma cacao (p 101) and the woodland strawberry Fragaria vesca ( $\mathrm{p}$ 109). But why are the sequences of these delicate, low yielding crop species valuable to geneticists? Firstly, the source of these plants' vulnerability is in both cases related to the genetic simplicity that provided the easy way in for the teams sequencing and assembling the genomes. Criollo cocoa became highly homozygous during its adaptation to human tastes at the cost of lower yields and disease susceptibility, traits that contrast with those of more robust derivative hybrids. Most large commercial strawberries are complex high polyploids much less amenable to de novo assembly of short sequence reads.

Secondly, relatively few plant species have yet been sequenced, but most of those that are now available are apt for the parallel study of mechanisms of evolution, both the mechanisms of speciation and those operating in adaptation at the population level in the course of domestication history and agricultural improvement. For example, the strawberry is a key to the genome evolution of the economically important Rosaceae, apple (Nat. Genet. 42, 833-839, 2010), rose, cherry and peach. No free lunch is on the table for all genome researchers, as some unusual model plants with no economic application may be needed to understand ecological adaptations, particularly those to extreme environments. We will consider those projects on their merits, assessing the likelihood that these model systems will influence future research and plant breeding.
Finally, the genomes are a treasure trove of biosynthetic pathways we ourselves lack and which we crave to complement from our favorite crops. Cocoa is extraordinarily rich in flavonoid nutrients and floral scented monoterpenes. The reference genomes this journal is interested in are those that rapidly lead to much more sequencing to uncover and use the available genome diversity in translational plant research to optimize disease resistance, yield and consumer qualities.

Genome projects considered by the journal will be required to be of high quality and transparency, with sequence reads and scaffolds available to referees and readers alike. The emphasis of the journal remains focused on genetic mechanisms studied by experimental perturbation of gene networks and by comparison and mapping of the traits of variants. You will find many examples in this issue alone, but the one to complement the chocolate and strawberries is the example of two-rowed barley. This cereal-another treat species traditionally favored by English and German brewers for its relatively high carbohydrate content-under agricultural selection evolved into higher-protein feed varieties that have six rows of grains. On page 169, Robbie Waugh and colleagues show that this distinction owes its origin to selection at the barley ortholog of the TEOSINTE $B R A N C H E D 1$ gene that was also selected by human hands for inflorescence adaptation during maize domestication. So, despite the recent flush of new first reference sequences of a forest of plants, we remain at heart a journal of genetic variation. 